\title{
Effects and prevalence of responders after a multicomponent intervention on cardiometabolic risk factors in children and adolescents with overweight/obesity: Action for health study
}

Caroline Brand, Clarice Maria De Lucena Martins, Vanilson Batista Lemes, Maria Luisa Félix Pessoa, Arieli Fernandes Dias, Eduardo Lusa Cadore, Jorge Mota, Adroaldo Cezar Araujo Gaya \& Anelise Reis Gaya

To cite this article: Caroline Brand, Clarice Maria De Lucena Martins, Vanilson Batista Lemes, Maria Luisa Félix Pessoa, Arieli Fernandes Dias, Eduardo Lusa Cadore, Jorge Mota, Adroaldo Cezar Araujo Gaya \& Anelise Reis Gaya (2020): Effects and prevalence of responders after a multicomponent intervention on cardiometabolic risk factors in children and adolescents with overweight/obesity: Action for health study, Journal of Sports Sciences, DOI: 10.1080/02640414.2020.1725384

To link to this article: https://doi.org/10.1080/02640414.2020.1725384

Published online: 12 Feb 2020.

Submit your article to this journal $\sqsubset$

View related articles ¿

View Crossmark data ¿ 


\title{
Effects and prevalence of responders after a multicomponent intervention on cardiometabolic risk factors in children and adolescents with overweight/obesity: Action for health study
}

\author{
Caroline Brand ${ }^{a}$, Clarice Maria De Lucena Martins ${ }^{b}$, Vanilson Batista Lemes (D) $^{a}$, Maria Luisa Félix Pessoa ${ }^{b, c}$, \\ Arieli Fernandes Dias ${ }^{\mathrm{a}}$, Eduardo Lusa Cadore ${ }^{\mathrm{d}}$, Jorge Mota ${ }^{\mathrm{D}}{ }^{\mathrm{e}}$, Adroaldo Cezar Araujo Gaya and Anelise Reis Gaya ${ }^{\mathrm{a}}$
}

aProjeto Esporte Brasil (PROESP-Br). School of Physical Education, Physiotherapy and Dance, Post-graduation Program in Human Movement Sciences, Federal University of Rio Grande do Sul, Porto Alegre, Brazil; 'besearch Center on Physical Activity, Health and Leisure, Federal University of Paraiba, João Pessoa, Brazil; cHealth Science Centre, Federal University of Paraíba, João Pessoa, Brazil; dSchool of Physical Education, Physiotherapy and Dance, Post-graduation Program in Human Movement Sciences, Federal University of Rio Grande Do Sul, Porto Alegre, Brazil; eResearch Center on Physical Activity, Health and Leisure (CIAFEL), Faculty of Sport, University of Porto, Porto, Portugal

\section{ABSTRACT}

This study aimed to verify the effect of a multicomponent intervention on cardiometabolic risk factors (CMRF), and to determine the prevalence of responders on CMRF among children and adolescents with overweight/ obesity. This is a quasi-experimental study, developed with 35 children and adolescents with overweight/ obesity (control group $(C G)=18$; intervention group $(I G)=17$ ), aged between 7 and 13 years. Participants in IG underwent a multicomponent intervention for 12 weeks. The following variables were evaluated: anthropometric measures, maturational stages and CMRF (body fatness, HOMA-IR, triglycerides, high-density and low-density lipoprotein) (HDL-C, LDL-C), total cholesterol (TC), aspartate aminotransferase (AST), alanine aminotransferase (ALT) and AST/ALT ratio. Mixed analysis of variance and the prevalence of responders were used for statistical analysis. There was a significant time $\mathrm{x}$ group interaction on body fatness $(p<0.001)$, HOMA-IR $(p=0.01)$, HDL-C $(p<0.001)$, LDL-C $(p=0.009)$ and TC $(p<0.001)$. The prevalence of responders for CMRF in IG and CG was respectively: body fatness (47\%; $0 \% ; p=0.04)$, HOMA-IR $(58.8 \% ; 16.6 \%$; $\mathrm{p}=0.04)$; triglycerides $(17.6 \% ; 5.5 \% ; \mathrm{p}=0.31) ; \mathrm{HDL}-\mathrm{C}(76.4 \% ; 5.5 \% ; \mathrm{p}=0.01), \mathrm{LDL}-\mathrm{C}(35.3 \% ; 5 \% ; \mathrm{p}=0.08), \mathrm{TC}$ (64.7\%; 5\%; $p=0.01)$, AST (5.8\%; 0\%; $p=0.87)$, ALT (29.4\%; $11.1 \% ; p=0.24)$ and AST/ALT ratio $(24.4 \% ; 22.2 \%$; $\mathrm{p}=0.67)$. Multicomponent intervention induced positive changes on CMRF along with a higher prevalence of positive adaptations in IG than the CG in some of the cardiometabolic outcomes assessed.
ARTICLE HISTORY

Accepted 14 January 2020

\section{KEYWORDS}

Obesity; physical exercise; youth; cardiometabolic health

\section{Introduction}

Paediatric obesity has been reached alarming proportions worldwide, resulting in adverse effects on physical and mental health (Hardy et al., 2017; Sahoo et al., 2015). Furthermore, childhood obesity has been associated with long-term problems, such as increased risk for the development of type 2 diabetes and cardiovascular disease (Fang et al., 2019). This scenario calls for the implementation of strategies for the treatment and prevention of obesity in early ages and multidisciplinary programmes are considered the most suitable approach (Ells et al., 2018).

It is also important to consider that obesity is associated with the development of a wide range of cardiometabolic risk factors (CMRF) (Chang et al., 2015). It has been described that high-density lipoprotein cholesterol (HDL-C) and low-density lipoprotein cholesterol (LDL-C) are strong and independent predictors of cardiovascular events (Toth et al., 2013). In addition, high triglycerides (TG) level is a marker for several types of atherogenic lipoproteins and a prevalent risk factor for cardiovascular disease (Talayero \& Sacks, 2011). In this context, an alteration of the profile corresponding to abnormal blood levels of TG and/or cholesterol-carrying lipoproteins affecting one or all of the subfractions of blood lipids is characterised as dyslipidemia. Insulin resistance (IR) is also an important metabolic disorder associated with obesity and diabetes mellitus (Yin et al., 2013). These metabolic abnormalities in children may indicate that cardiovascular disease complications appear at early ages and facilitate the development of risk factors for cardiovascular disease in adulthood (Chang et al., 2015).

Moreover, Non-Alcoholic Fatty Liver Disease (NAFLD) is another health problem related to all above-mentioned risk factors (Alkhater, 2015). Previous studies have reported that liver enzymes, such as aspartate aminotransferase (AST) and alanine aminotransferase (ALT) are markers of NAFLD among children and adolescents with obesity (Draijer et al., 2019; Vos et al., 2017). Also, AST/ALT ratio has been used as a marker of NAFLD (Alkhouri \& McCullough, 2012).

In this context, many systematic reviews and experimental studies have been describing the effect of multicomponent lifestyle interventions, that included physical exercise on CMRF in youth. Escalante, Saavedra, Garcia-Hermoso, and Dominguez (2012) and Fedewa, Gist, Evans, and Dishman (2014) indicated that exercise training is effective on improving HDL-C, LDL-C and TG concentrations, as well as IR in children and adolescents. In addition, a lifestyle intervention of 6 months was effective in improving NAFLD markers and IR in 
severely children with obesity (Koot et al., 2011). Moreover, a recent evidence-based exercise recommendation to reduce hepatic fat content showed that exercise training is an important strategy to improve and prevent hepatic steatosis in the paediatric population (Medrano et al., 2018).

Findings regarding the benefits of physical activity on health in youth have been widely shown. However, studies on this issue are mainly focused on analysing it in terms of "mean". Notwithstanding, it has been shown that there is a wide interindividual variability in the effects of exercise interventions in health outcomes (Bonafiglia et al., 2016). It means that, under the same stimulus, while some individuals may achieve benefits after training (responders), other can present an unchanged or worsened response (non-responders) (Bonafiglia et al., 2016). According to Bouchard and Rankinen (2001) even when subjects are exposed to the same volume of physical activity, there are considerable individual differences in the response to exercise, at least concerning risk factor changes. Those aspects have been investigated in adults, indicating that the CMRF responses to exercise training, such as cardiorespiratory fitness, fasting insulin and glucose, have not been fully clarified (Álvarez, RamírezCampillo, Ramírez-Vélez, \& Izquierdo, 2017a; Bouchard et al., 2012; Montero \& Lundby, 2017). On the other hand, this interindividual variability has been little explored in youth individuals.

The literature regarding interindividual variability in the adaptations to exercise in health outcomes is limited in children and adolescents (Alvarez, Ramírez-Campillo, RamírezVélez, \& Izquierdo, 2017b), especially considering a wide range of cardiometabolic risk factors and a multicomponent intervention. Also, we have evaluated children and adolescents of low-income status because some studies have shown a higher IR, a more adverse lipid profile and high rates of overweight among the lowest socioeconomic groups (Hauger et al., 2016; WHO, 2017).

Therefore, the aims of the present study were (1) to verify the effect of a multicomponent intervention on CMRF, and (2) to determine the prevalence of responders after the multicomponent intervention on CMRF among children and adolescents with overweight/obesity. We hypothesised that this sample will present a high prevalence of responders on cardiometabolic risk factors following a multicomponent intervention programme.

\section{Methods}

\section{Study design}

This is a quasi-experimental study, part of the "ACTION FOR HEALTH" (Adolescents and Children in a Training Intervention for Health) project, developed with low-income children and adolescents with overweight/obesity of both genders, aged between 7 and 13 years. This multicomponent intervention program includes physical activity after-school classes, nutritional education sessions, and parental support. The project was developed in 2015, during a 12-week period. All the procedures were approved by the Scientific Board of the Research Unit that leads the project (excluded information for blind review), and is registered in www.clinicaltrials.gov (Number: NCT02929472). Also, all the Helsinki Declarations' ethical aspects were followed (World Medical Association, 2013). The parents from those who agreed to participate signed the consent form as well as the children and adolescents signed the assent form. More detailed information about the study design and intervention process was published before (Silveira et al., 2018).

\section{Participants and procedures}

This project was publicised in two educational poles (six public schools) from João Pessoa-PB (Brazil), which were located in regions nearby the intervention site. All schools were located in a deprived area, with low socioeconomic status: $62.5 \%$ of the mothers or fathers were unemployed and over $45 \%$ of the mothers and $64 \%$ of the fathers had concluded the ninth grade or less. Parents of potential participants ( $n=276$, according to school teacher's or parent's information) were invited to participate in early evening information meetings with the multidisciplinary staff. From those, 96 parents attended the meetings, where all the potential participants were screened for inclusion criteria, defined as having overweight or obesity (Cole, Bellizzi, Flegal, \& Dietz, 2000). From those, 53 were excluded, leaving 43 in the study, which were allocated by convenience in the control group (CG, $n=20)$ and intervention group ( $G, n=23)$. However, 2 participants did not complete baseline evaluation in CG, and 6 in IG, leaving a total of 17 participants in CG and 18 in IG (Figure 1). Thus, all participants that completed evaluations at baseline were submitted to the intervention and there were no losses after the 12 weeks. Also, Children and parents of the control group were advised to do not change their lifestyle during the period between evaluations.

\section{Multicomponent intervention program}

The multicomponent intervention program was developed during 12 weeks, consisting of exercise sessions (twice/week; $1 \mathrm{~h})$, nutritional education sessions (once/month), and parental support (twice/week). The program was developed by four physical education teachers, one nutritionist, and one paediatrician, who previously participated in weekly meetings (over 6 months), which methodologies and intervention sessions were prepared and tested. Two graduate physical education students and two graduate nutrition students collaborated in the program weekly sessions during all the intervention program. All the staff was trained and supervised by the project coordinator.

\section{Physical exercise intervention}

The exercise sessions took 60 min, twice a week, for 12 weeks, always taught by the same physical education teachers. Each session included 10 min of warm-up; 30 min of circuit training; $15 \mathrm{~min}$ of pre-sports and recreational games; and $5 \mathrm{~min}$ of resting activities. The activities were planned so that the children could maintain a moderate to vigorous intensity throughout the class. Warm-up included aerobic/anaerobic and recreational activities. The circuit was organised into six stations. The focus of each station was the same during all the intervention program and included activities that prioritised physical fitness capacities (Weineck, 2005), for example, rarely 


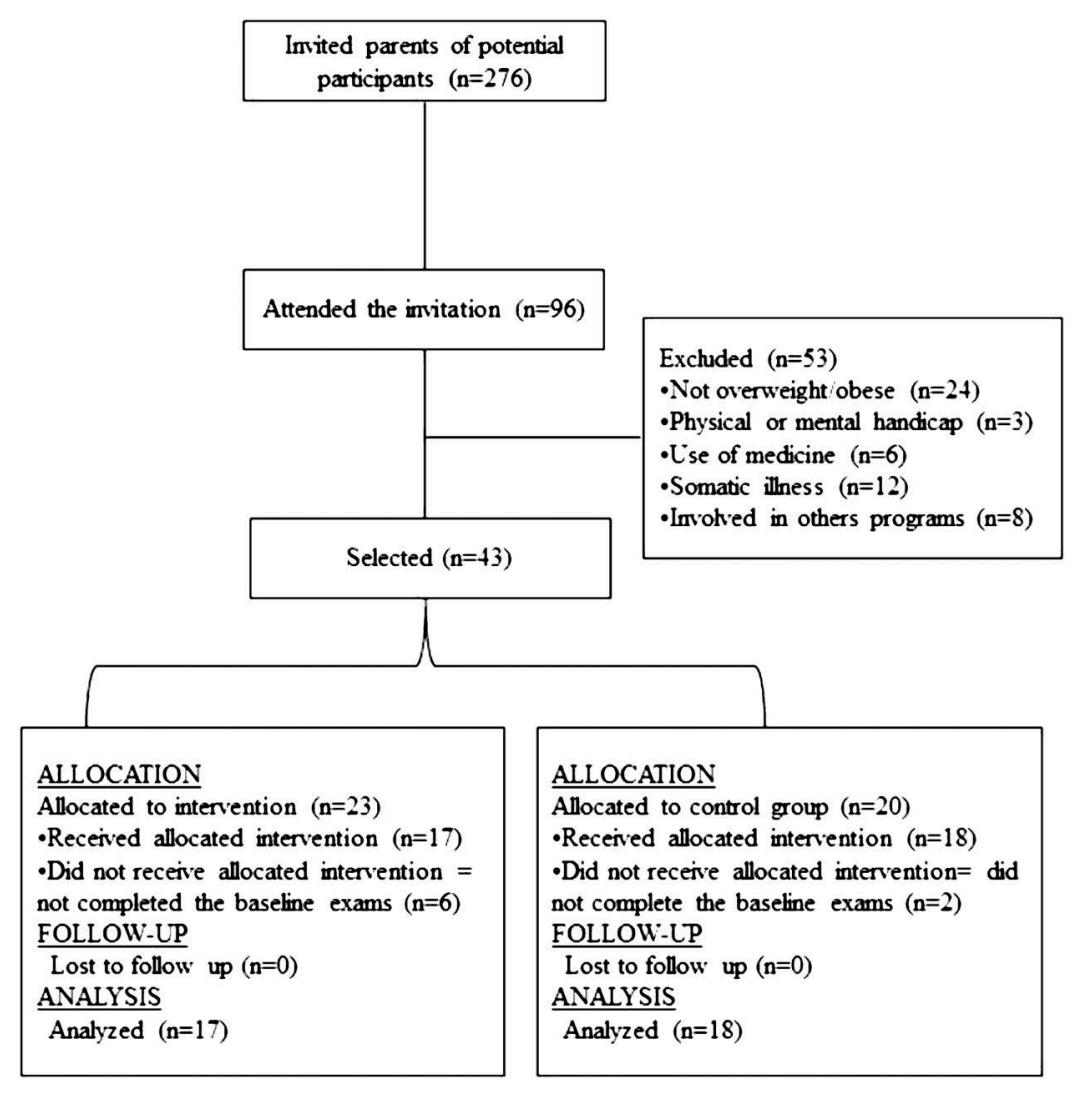

Figure 1. Sample's flow diagram.

race, jump rope, throw different size balls training warm-up, high-knee skipping dribbling a ball, climb a rope, jumping jacks in unstable platform. The activities developed in each station were modified to ensure children's motivation and adherence. Exercises were structured so that participants could maintain a high intensity throughout the circuit. This protocol has been used in other intervention studies with a similar sample (Aires et al., 2016; Nascimento et al., 2014).

Training intensity and compliance between individuals were defined to induce heart rate (HR) higher than $65 \%$ of $\mathrm{HR}_{\max }$ of each participant. To monitor the activity, 10 randomly selected participants wore a portable HR monitor (Polar Team ${ }^{2}$ Pro, Polar, Finland) during sessions. Attendance average for IG was $85 \%$ (minimum of $73.6 \%$ and maximum of $88.9 \%$ ).

\section{Nutritional education intervention}

The dietary intervention, designed by the nutritionist staff, consisted of two actions developed in groups for children and parents: 1) three events (one per month) for dietary counselling with parents, based on the recorded-dietary evaluation of their children. The attendance to counselling appointments was $100 \%$; 2) 3 daily dietary goals for participants. These goals were based on three main points: frequency and quantity of adequate and inadequate food, and water consumption (i.e., replace the white rice with brown rice in at least one meal a day; drink milk without chocolate or sugar at least once a day). Participants received a worksheet to record whether they met the daily goals and all participants adhered to the completion of the dietary sheets. No significant inter-subject variance was observed for the achievement of the proposed goals in the IG (not presented).

\section{Parental support intervention}

Simultaneously, exercise sessions were offered to all parents in order to encourage family support. Adults' participation was voluntary and the parental support intervention was focused on improvement of 3 aspects: (a) encouragement through provision of transportation to PA facilities (100\% attendance); (b) participation in PA with the children, when applicable $(82 \%$ attendance); (c) watching the children during physical activities (95\% attendance). The activities were carried out by a trained physical education teacher in the centre of sports of the Federal University of Paraíba, João Pessoa (Brazil), during children's same schedule. Parent's attendance average for the three different actions was $88 \%$.

\section{Measurements}

Baseline measurements were performed during a 14-day period, for CG and IG groups. On the first day, the blood collection was taken, anthropometric outcomes were assessed and maturational stages were determined; on the second day, body fatness was determined. Anthropometric variables, maturational stages and body fatness evaluation were carried out at the Physical Education Department of the Federal University of Paraiba. The blood analysis was carried out at a convened specialised laboratory in João Pessoa/Brazil. For post-intervention evaluation, the same procedures were followed by the same blind staff. All the 
post-intervention data were collected during the subsequent week after the intervention.

\section{Anthropometric measures and body composition}

Height and weight were determined by a "Holtain" stadiometer, then body mass index was calculated, by dividing body mass (in kilograms) by height (in square metres). Children and adolescents were classified as overweight/obesity according to Cole et al. (2000). Body fatness was measured after 4-h-fasting and low water intake with a bioimpedance scale (Inbody 720, Biospace Co. Ltd.). Those measures were taken following standardised procedures (Lohman, Roche, \& Martorell, 1988). To determine waist circumference, the NHANES protocol (US Department of Health and Human Services, 1996) was followed.

\section{Maturational stages}

The breast stages (B1-B5) in girls and the genital stages (G1-G5) in boys were assessed according to Tanner's criteria [31] by a paediatrician at the first day of measurements.

\section{Biochemical assays}

The CMRF considered in the present study were: body fatness, homoeostasis model assessment of insulin resistance (HOMAIR), TG, HDL-C, LDL-C, total cholesterol (TC), AST, ALT, AST/ALT ratio. The circulating levels of plasma insulin, glucose, $T G$, cholesterols (TC and HDL-C), as well as serum AST and ALT were measured through peripheral puncture in the cubital vein after a nocturnal 12-hour-fasting, by laboratory specialists, using standard techniques at an accredited partner laboratory. The analysis of TC, HDL-C, TG, and glucose was carried out by spectrophotometry (Cobas Integra 400 Plus) with Roche ${ }^{\circledR}$ kits. The LDL-C fraction was indirectly calculated using the Friedewald formula (Friedewald, Levy, \& Fredrickson, 1972). The AST and ALT levels were determined by enzyme kinetic assay for spectrophotometrically obtained after 10 min centrifugation at $3500 \mathrm{rpm}$. Thus, it was calculated the AST/ALT ratio. Insulin was determined by Luminex-100 IS (Integrated System: Luminex Corporation, Austin, TX, USA), using the Linco Human Gut Hormone panel kit (Linco Research Inc., MO, USA). As a proxy measure of IR, HOMA-IR was calculated as the product of basal glucose $(\mathrm{mmol} / \mathrm{L})$ and insulin $(\mu \mathrm{lU} / \mathrm{mL})$ levels divided by 22.5 (Matthews et al., 1985). All samples were run in duplicates and the means were calculated. The intra-assay coefficients of variation (CV) were less than $20 \%$, according to the manufacturer.

\section{Statistical analysis}

Descriptive procedures were performed for all independent and dependent variables, through means and confidence intervals. One-way ANOVA test was used to compare baseline variables, between CG and IG. The intervention effect on time, group and interaction (time $x$ group) were verified through mixed ANOVA, adjusted for age, sex and sexual maturation.

Prevalence of responders in the outcome variables in CG and IG was obtained according to the theoretical model applied in previous studies with biochemical variables outcomes, considering the $\Delta \%$ effect (\% of the after minus the before value) (Álvarez, Ramírez-Campillo, \& Ramírez-vélez, 2017c; Álvarez et al., 2017a, 2018). However, due to the impossibility of two basal measures in each outcome variable in the present study, we did not calculate the reliability intrasubject (typical error) as suggested to this kind of analysis (Alvarez et al., 2017b; Álvarez et al., 2017a; Hopkins, 2000). To minimise this limitation, we considered as acceptable clinical and individual effects, the values of $\Delta \%$ obtained from previous interventions with the same variables as parameters and cut-points to responders of the intervention (R). The cut-points for body fatness (change of the cut-points $R>16 \%$ ), was based on (Reinehr et al., 2010). HDL-C (change of the cut-points $4.2 \mathrm{mg} / \mathrm{dL}, \mathrm{R}>10 \%$ ), LDL-C (change of the cut-points $-12.4 \mathrm{mg} / \mathrm{dL}, \mathrm{R}>10 \%$ ), TG (change of the cut-points $-10.1 \mathrm{mg} / \mathrm{dL}, \mathrm{R}>10 \%$ ) and TC (change of the cutpoints $-7.7 \mathrm{mg} / \mathrm{dL}, \mathrm{R}>5 \%$ ) were based on Escalante et al. (2012), while Koot et al. (2011) was considered as reference for AST (change of the cut-points $-8.4 \mathrm{U} / \mathrm{L}, \mathrm{R}>26 \%$ ), ALT (change of the cut-points $-5.6 \mathrm{U} / \mathrm{L}, \mathrm{R}>20 \%$ ), AST/ALT ratio (change of the cut-points $-0.10 \mathrm{U} / \mathrm{L}, \mathrm{R}>9 \%$ ), and HOMA-IR (change of the cut-points $-1.0, \mathrm{R}>30 \%$ ).

Finally, to verify the difference in the prevalence of responders between IG and CG, Poisson regression was applied in generalised estimative equation. All the analyses were carried out using the IBM SPSS 21 (SPSS, Inc., Chicago, Illinois, USA). The level of statistical significance was established as $p<0.05$.

\section{Results}

Characteristics for all variables in both groups at baseline are presented in Table 1. There were no differences in the variance between groups in any of the variables analysed at baseline. The sample was composed of $37.1 \%$ of boys and $62.9 \%$ of girls.

Table 1. Sample characteristics and comparison between control and intervention group at baseline.

\begin{tabular}{|c|c|c|c|c|c|c|}
\hline & \multicolumn{2}{|c|}{ Control Group $(n=18)$} & \multicolumn{2}{|c|}{$\begin{array}{l}\text { Intervention Group } \\
\qquad(\mathrm{n}=17)\end{array}$} & \multicolumn{2}{|c|}{ Anova } \\
\hline & Mean & $95 \% \mathrm{Cl}$ & Mean & $95 \% \mathrm{Cl}$ & $\mathrm{F}$ & $p$ \\
\hline Age (years) & 8.27 & (7.42 9.12) & 8.17 & (7.06 9.41) & 0.01 & 0.89 \\
\hline Weight (kg) & 39.29 & (34.16 44.75) & 40.90 & (32.62 50.21) & 0.09 & 0.75 \\
\hline Height (m) & 1.34 & $(1.29$ 1.38) & 1.34 & $(1.271 .41)$ & 0.00 & 0.98 \\
\hline BMI $\left(\mathrm{kg} / \mathrm{m}^{2}\right)$ & 21.88 & (20.28 23.52) & 21.97 & (19.57 24.54) & 0.00 & 0.95 \\
\hline $\begin{array}{l}\text { Body fatness } \\
(\%)\end{array}$ & 14.13 & (11.04 17.38) & 13.60 & (8.97 18.67) & 0.03 & 0.85 \\
\hline$W C(\mathrm{~cm})$ & 73.81 & (68.58 78.95) & 71.26 & (64.30 78.58) & 0.32 & 0.57 \\
\hline Insulin (UI/ml) & 6.30 & (5.24 7.61) & 9.09 & (6.85 11.67) & 4.15 & 0.05 \\
\hline $\begin{array}{l}\text { Glucose } \\
\text { (mg/dL) }\end{array}$ & 86.27 & (82.95 89.85) & 83.12 & (80.74 85.41) & 2.17 & 0.15 \\
\hline HOMA-IR & 1.36 & (1.09 1.68) & 1.88 & (1.41 2.41) & 3.01 & 0.09 \\
\hline TG (mg/dL) & 80.63 & (68.53 94.19) & 76.05 & (63.31 89.72) & 0.23 & 0.63 \\
\hline $\mathrm{HDL}-\mathrm{C}(\mathrm{mg} / \mathrm{dL})$ & 50.61 & (44.98 56.52) & 46.00 & (42.15 49.75) & 1.65 & 0.20 \\
\hline LDL-C (mg/dL) & 94.49 & (82.91 106.73) & 97.93 & (89.99 106.41) & 0.20 & 0.65 \\
\hline $\mathrm{TC}(\mathrm{mg} / \mathrm{dL})$ & 158.11 & $\begin{array}{l}(143.47 \\
172.27)\end{array}$ & 171.64 & $\begin{array}{r}(162.98 \\
181.07)\end{array}$ & 2.28 & 0.14 \\
\hline AST (U/L) & 26.39 & (23.82 29.45) & 28.65 & (25.13 32.13) & 0.98 & 0.32 \\
\hline ALT (U/L) & 18.95 & (16.85 21.54) & 19.53 & (18.20 20.94) & 0.16 & 0.68 \\
\hline AST/ALT ratio & 1.44 & $(1.281 .66)$ & 1.48 & (1.31 1.65) & 0.08 & 0.77 \\
\hline
\end{tabular}

BMI: Body mass index; WC: Waist circumference; HOMA-IR: Homoeostasis model assessment of insulin resistance; TG: Triglycerides; HDL-C: High-density lipoprotein cholesterol; LDL-C: low-density lipoprotein cholesterol; TC: Total cholesterol; AST: aspartate aminotransferase; ALT: alanine aminotransferase. 
The prevalence of children and adolescents with overweight was $65.7 \%(n=23)$ and $34.3 \%(n=12)$ with obesity. For girls, the prevalence of overweight was $76.9 \%$ and $59.1 \%$ for boys were overweight. Concerning pubertal stages, 38.9\% of the girls were in stage $\mathrm{B} 1,55.5 \%$ in $\mathrm{B} 2$ and $5.6 \%$ in B3, while for boys $50 \%$ were in $\mathrm{G} 1,33.3 \%$ in $\mathrm{G} 2$ and $16.7 \%$ in $\mathrm{G} 3$.

Table 2 indicates the effect of time, group and interaction on CMRF. It was found a significant interaction (time $x$ group) on body fatness, HOMA-IR, HDL-C, LDL-C, TC and ALT. Regarding AST, AST/ALT ratio and TG there was no significant effect of time, group and interaction.

Figures 2-4 showed the prevalence of responders on CMRF, after the multicomponent intervention for the IG and CG. It was found difference in the prevalence of responders between IG and CG in body fatness (IG $=47 \%$; CG $=0 \%$; PR (prevalence ratio $)=8.47 ; \mathrm{p}=0.04)$, HOMA-IR (IG $=58.8 \% ; \mathrm{CG}=16.6 \% ; \mathrm{PR}=$ 3.52; $\mathrm{p}=0.04), \mathrm{HDL}-\mathrm{C}(\mathrm{IG}=76.4 ; \mathrm{CG}=5.5 ; \mathrm{PR}=13.76 ; \mathrm{p}=0.01)$ and TC (IG $=64.7 \% ; C G=5 \% ; P R=11.64 ; p=0.01)$. For the other variables it was not observed differences between groups in the prevalence of responders, TG (IG $=17.6$; $\mathrm{CG}=5.5 \%$; $\mathrm{PR}=$ 3.17; $p=0.31)$, LDL-C $(I G=35.3 \% ; C G=5 \% ; P R=6.35 ; p=0.08)$, AST (IG = 5.8\%; CG =0\%; PR = 1.79; $p=0.87), A L T(I G=29.4 \%$;
$C G=11.1 \% ; P R=2.64 ; p=0.24)$ and $A S T / A L T$ ratio $(I G=24.4 \%$; $C G=22.2 \% ; P R=1.32 ; p=0.57)$.

\section{Discussion}

The main findings of this study suggest that: (1) the multicomponent intervention group showed positive changes in several CMRF such as body fatness, HOMA-IR, HDL-C, LDL-C, TC and ALT, while no positive changes were observed in the CG; and (2) the prevalence of responders of CMRF in IG was heterogeneous throughout the outcomes assessed: body fatness (47\%), HOMA-IR (58.8\%), TG (17.6\%), HDL-C (76.4\%), LDL-C (35.3\%), TC (64.7\%), AST (65.8\%), ALT (29.4\%) and AST/ALT ratio (24.4\%), suggesting that individual responses to intervention in youth depends on the cardiometabolic outcome assessed, showing high level of intervariability. It may be speculated that the heterogeneous prevalence of responders for each cardiometabolic risk outcome could be explained by the different cut-point used for the definition of responders; notwithstanding, the cut-points were established considering studies with similar characteristics to our study. Concerning prevalence of responders between groups, it was found

Table 2. Intervention effect in time, group and interaction on cardiometabolic risk factors.

\begin{tabular}{|c|c|c|c|c|c|c|c|c|c|c|c|c|c|}
\hline \multirow[b]{3}{*}{ Variables } & \multirow[b]{3}{*}{ Groups** } & & \multirow[b]{3}{*}{ Mean* } & \multirow[b]{3}{*}{ SE } & \multicolumn{9}{|c|}{ Mixed Anova* } \\
\hline & & & & & \multicolumn{3}{|c|}{ Time } & \multicolumn{3}{|c|}{ Group } & \multicolumn{3}{|c|}{ Time $x$ Group } \\
\hline & & & & & $\mathrm{F}$ & $p$ & Eta & $\mathrm{F}$ & $\mathrm{p}$ & Eta & $\mathrm{F}$ & $\mathrm{p}$ & Eta \\
\hline \multirow[t]{4}{*}{ Body fatness (\%) } & CG & pre & 16.18 & 2.02 & & & & & & & & & \\
\hline & & post & 17.06 & 1.92 & 1.50 & 0.23 & 0.05 & 3.06 & 0.09 & 0.10 & 16.95 & $<0.001$ & 0.38 \\
\hline & IG & pre & 12.36 & 1.82 & & & & & & & & & \\
\hline & & post & 11.42 & 1.73 & & & & & & & & & \\
\hline \multirow[t]{4}{*}{ HOMA-IR } & CG & pre & 1.43 & 0.25 & & & & & & & & & \\
\hline & & post & 1.85 & 0.22 & 0.25 & 0.61 & 0.00 & 0.03 & 0.85 & 0.00 & 7.06 & 0.01 & 0.20 \\
\hline & IG & pre & 1.80 & 0.22 & & & & & & & & & \\
\hline & & post & 1.38 & 0.20 & & & & & & & & & \\
\hline \multirow[t]{4}{*}{$\mathrm{TG}(\mathrm{mg} / \mathrm{dL})$} & CG & pre & 80.52 & 7.74 & & & & & & & & & \\
\hline & & post & 100.73 & 8.64 & 3.61 & 0.06 & 0.11 & 0.28 & 0.60 & 0.01 & 0.01 & 0.98 & 0.00 \\
\hline & IG & pre & 75.61 & 7.00 & & & & & & & & & \\
\hline & & post & 96.03 & 7.81 & & & & & & & & & \\
\hline \multirow[t]{4}{*}{$\mathrm{HDL}-\mathrm{C}(\mathrm{mg} / \mathrm{dL})$} & CG & pre & 48.71 & 2.90 & & & & & & & & & \\
\hline & & post & 43.72 & 2.80 & 0.008 & 0.92 & 0.01 & 2.02 & 0.16 & 0.07 & 30.58 & $<0.001$ & 0.53 \\
\hline & IG & pre & 47.05 & 2.62 & & & & & & & & & \\
\hline & & post & 55.94 & 2.53 & & & & & & & & & \\
\hline \multirow[t]{4}{*}{ LDL-C (mg/dL) } & CG & pre & 93.91 & 6.54 & & & & & & & & & \\
\hline & & post & 103.49 & 6.80 & 0.64 & 0.42 & 0.02 & 0.16 & 0.69 & 0.00 & 7.91 & 0.009 & 0.22 \\
\hline & IG & pre & 97.53 & 5.91 & & & & & & & & & \\
\hline & & post & 92.79 & 6.15 & & & & & & & & & \\
\hline \multirow[t]{4}{*}{$\mathrm{TC}(\mathrm{mg} / \mathrm{dL})$} & CG & pre & 157.98 & 7.78 & & & & & & & & & \\
\hline & & post & 168.33 & 8.10 & 0.08 & 0.77 & 0.00 & 0.04 & 0.95 & 0.00 & 19.07 & $<0.001$ & 0.41 \\
\hline & IG & pre & 171.54 & 7.03 & & & & & & & & & \\
\hline & & post & 156.06 & 7.23 & & & & & & & & & \\
\hline \multirow[t]{4}{*}{ AST (U/L) } & CG & pre & 25.23 & 1.70 & & & & & & & & & \\
\hline & & post & 26.58 & 1.37 & 1.40 & 0.24 & 0.05 & 0.27 & 0.60 & 0.01 & 3.40 & 0.07 & 0.11 \\
\hline & IG & pre & 29.57 & 1.53 & & & & & & & & & \\
\hline & & post & 27.00 & 1.24 & & & & & & & & & \\
\hline \multirow[t]{4}{*}{ ALT (U/L) } & CG & pre & 17.58 & 1.16 & & & & & & & & & \\
\hline & & post & 22.54 & 3.79 & 3.37 & 0.07 & 0.11 & 1.41 & 0.24 & 0.05 & 7.64 & 0.01 & 0.22 \\
\hline & IG & pre & 19.64 & 1.05 & & & & & & & & & \\
\hline & & post & 17.06 & 3.43 & & & & & & & & & \\
\hline \multirow[t]{4}{*}{ AST/ALT ratio } & CG & pre & 1.47 & 0.09 & & & & & & & & & \\
\hline & & post & 1.44 & 0.11 & 1.47 & 0.23 & 0.05 & 1.03 & 0.31 & 0.03 & 0.99 & 0.32 & 0.03 \\
\hline & IG & pre & 1.52 & 0.08 & & & & & & & & & \\
\hline & & post & 1.64 & 0.10 & & & & & & & & & \\
\hline
\end{tabular}

F: Mixed Anova; p: significance level <0.05; Eta: eta squared effect; CG: control group; IG: intervention group; SE: standard error; TG: Triglycerides; HDL-C: High-density lipoprotein cholesterol; LDL-C: low-density lipoprotein cholesterol; TC: Total cholesterol; AST: aspartate aminotransferase; ALT: alanine aminotransferase; HOMA: Homoeostasis model assessment of insulin resistance; *adjusted for age, sex and sexual maturation; ${ }^{* *} \mathrm{CG} n=17$ and IG $\mathrm{n}=18$. 

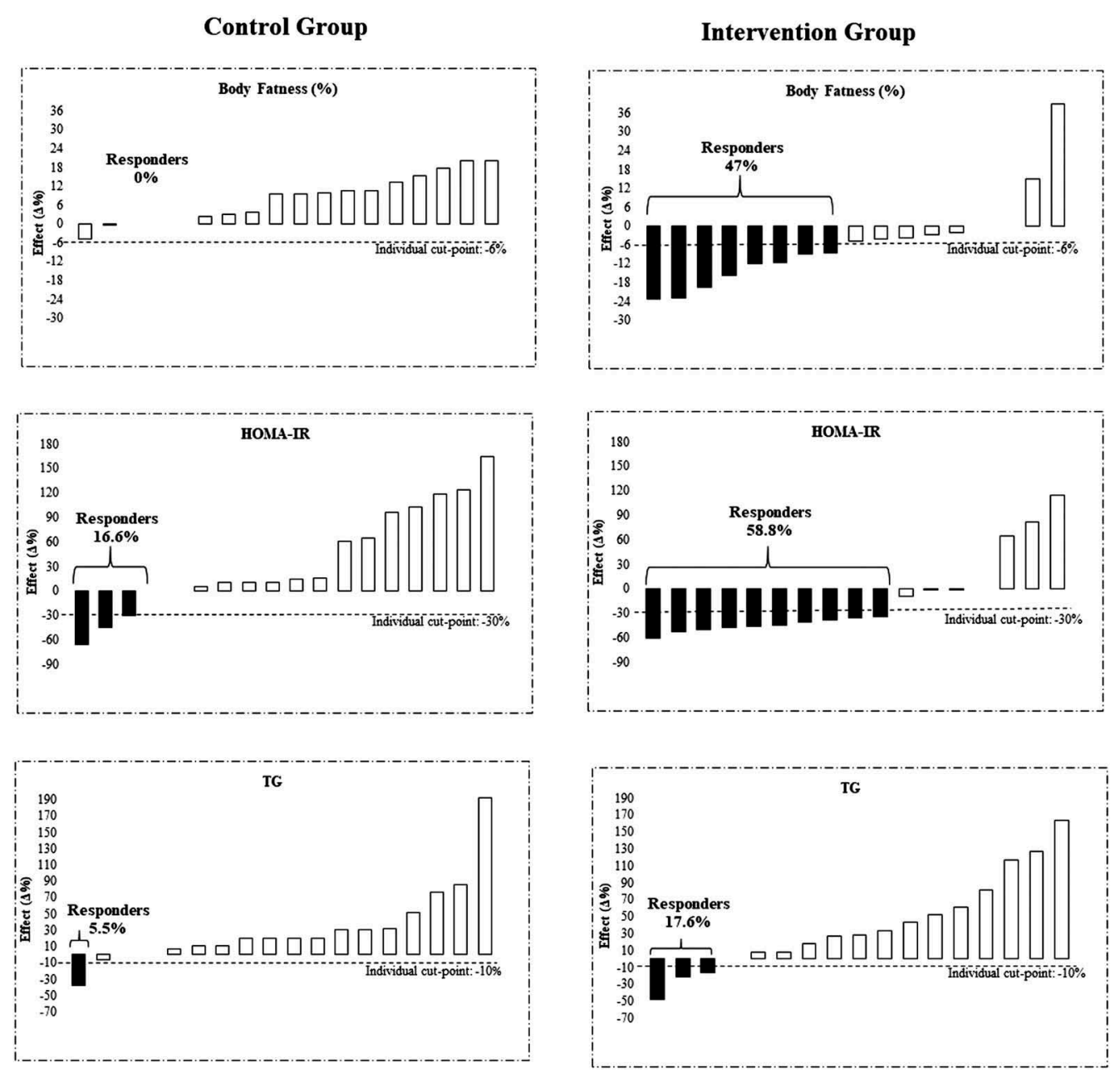

Figure 2. Prevalence of responders in intervention and control group after a multicomponent intervention for body fatness, homoeostasis model assessment of insulin resistance (HOMA-IR) and triglycerides (TG). CG $n=18$ and IG $n=17$. *difference in the prevalence ratio between groups.

differences in body fatness $(\mathrm{RP}=8.47 ; \mathrm{p}=0.04)$, HOMA-IR $(\mathrm{PR}=$ 3.52; $p=0.04)$, HDL-C (PR = 13.76; $p=0.01)$ and TC $(P R=11.64$; $p=0.01)$. These findings suggest that youth in IG improved CMRF after a multicomponent intervention, comparatively to those in CG, which worsened these parameters.

The effect of the intervention considering mean values showed that besides the variables that showed difference in the prevalence of responders between groups (body fatness, HOMA-IR, TC and HDL-C), other variables presented the effect of the intervention (LDL-C and ALT). Considering the results in terms of mean, previous studies have shown similar results. For instance, Resaland, Nilsen, Bartholomew, Andersen, and Anderssen (2018) showed that a two-year school-based daily physical activity intervention was able to decrease TG, as well as other cardiometabolic risk factors. Regarding NAFLD parameters, in accordance with our findings, Africa, Newton, and Schwimmer (2016) found a decrease of ALT concentration in youths at risk profiles after lifestyle interventions; the studies included in this review had the durations ranging from 4 weeks to 1 year. The positive results regarding lipid profile were also observed in a systematic review and meta-analysis, indicating that lifestyle interventions are effective in decreasing levels of
LDL-C, TG and fasting insulin in children with obesity after 4 weeks to 2 years of intervention (Ho et al., 2012).

Thus, our results reinforce the literature data concerning the effects of multicomponent intervention in the cardiometabolic risk outcomes in children and adolescents with overweight/ obesity, showing that nutritional education and parental support may positively contribute along with exercise intervention in order to improve these outcomes in the participants. Also, our data highlight that 12 weeks of intervention is enough to represent an improvement in overall CMRF in children and adolescents with overweight/obesity. This findings are especially relevant taken into consideration the high prevalence of obesity, as well as the increasing occurrence of risk to the cardiometabolic and musculoskeletal health in recent years in Brazilian children and adolescents (Flores, Gaya, Petersen, \& Gaya, 2013; Gaya et al., 2017).

According to our knowledge, there is no evidence regarding the prevalence of responders on cardiometabolic risk after a multicomponent intervention composed by exercise, nutritional education and family support in children and adolescents with overweight/obesity. Some studies have described the effect of intervention in terms of "mean". Meyer, Kundt, 
Control Group
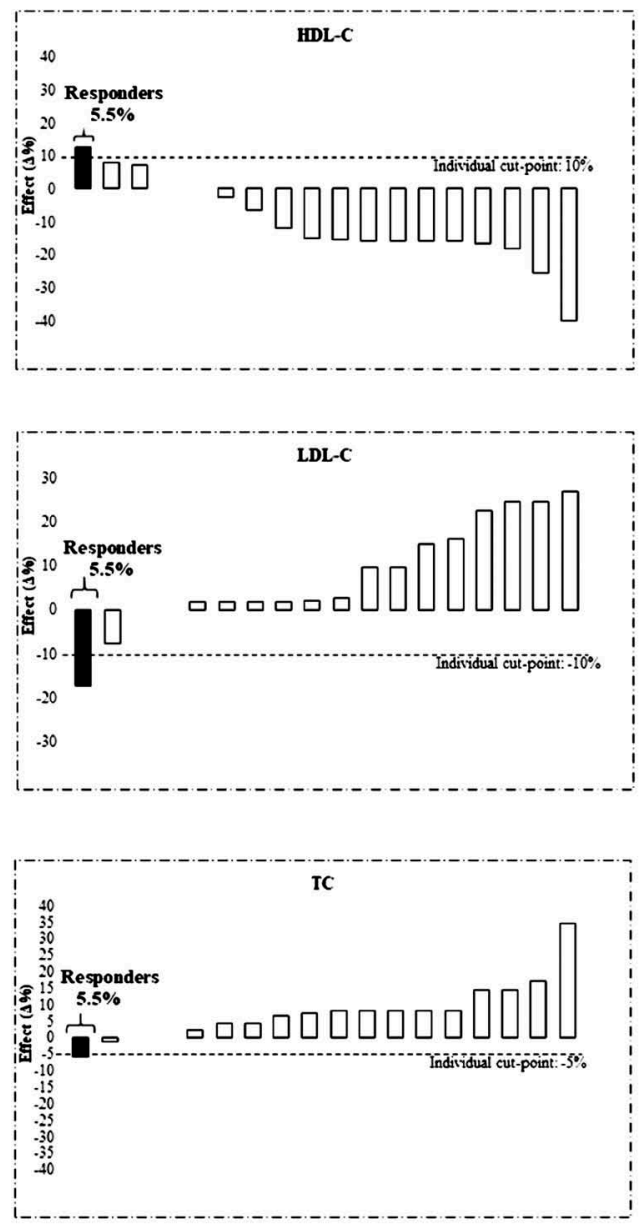

Intervention Group
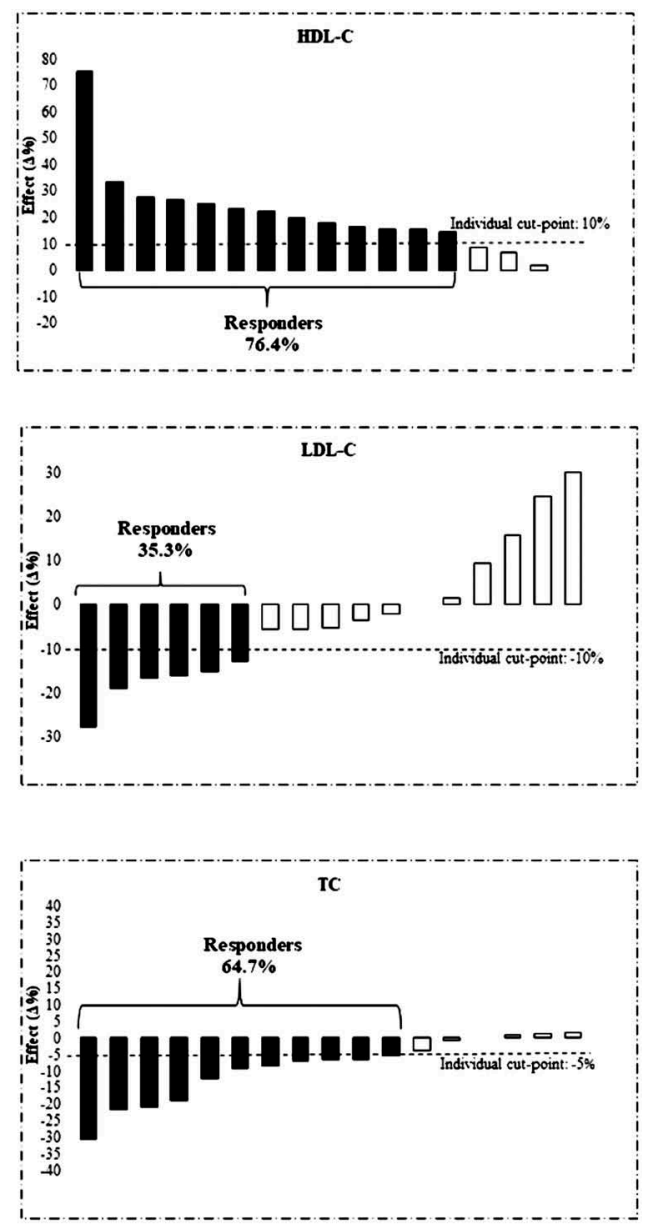

Figure 3. Prevalence of responders in intervention and control group after a multicomponent intervention for high-density lipoprotein cholesterol (HDL-C), low-density lipoprotein (LDL-C) and total cholesterol (TC). (CG $n=18$ and IG $n=17)$. ${ }^{*}$ difference in the prevalence ratio between groups.

Lenschow, Schuff-werner, and Kienast (2006) showed reductions of $3 \%$ in body fatness, $5 \%$ in LDL-C, $26 \%$ in HOMA-IR and $23 \%$ in TG after a 6-month exercise program in children with obesity. In the same population, Karacabey (2009) found improvements of $13 \%$ in HDL-C and reductions of $29 \%$ in LDL-C after 12 months of aerobic exercise program. Regarding NAFLD parameters, a 6-month lifestyle intervention for children presented reductions of $24 \%$ in ALT and $36 \%$ in AST (Koot et al., 2011). However, these authors did not report the individual prevalence of responsiveness among individuals. Considering that people exhibit a specificity of response, inducing a wide interindividual variability in the adaptations to exercise training, we aimed to verify the individual prevalence of responsiveness on different CMRF after a multicomponent intervention. Our findings indicated a prevalence of responders on body fatness (47\%), HOMA-IR (58.8\%), TG (17.6\%), HDL-C (76.4\%), LDL-C (35.3\%), TC (64.7\%) AST (5.8\%), ALT (29.4\%) and AST/ ALT ratio (24.4\%) in IG, and significant different prevalence between groups in body fatness, HOMA-IR, HDL-C, TC. These findings indicate that the prevalence of responders depends on the cardiometabolic risk outcome assessed. Moreover, characteristics of training, such as volume, and exercise intensity (Kraus, 2002) have an effect on exercise-induced changes in blood lipids, and it seems that HDL-C is the most sensitive to exercise (Wang \& Xu, 2017), which is in accordance with our findings.

In this line, a study developed with schoolchildren investigated the prevalence of non-responders, regarding different cardiometabolic and anthropometric indicators, after a highintensity interval training and found that the prevalence of non-responders in a group of early maturation was $25 \%$ for HOMA-IR and 35\% in normal maturation (Alvarez et al., 2017b). Although the multicomponent intervention developed in the present study had different methodological characteristics, the prevalence of individual that did not presented changes in HOMA-IR, was similar, i.e., approximately $41.2 \%$. Álvarez et al. (2018) found a lower prevalence of non-responders for HOMAIR following a high-intensity interval training $(17 \%)$, or a resistance training (18.5\%); However, those children were diagnosed with IR (Álvarez et al., 2018), which was not the case in the present study. Some studies with adults, using different types of exercise, have reported the prevalence of non-responders. Álvarez et al. (2017c) showed that the prevalence of non-responders for HOMA-IR was $25 \%$ for woman with high IR and $45 \%$ for those with low IR, after a high-intensity interval training. Besides, after a high-intensity interval training 
Control Group
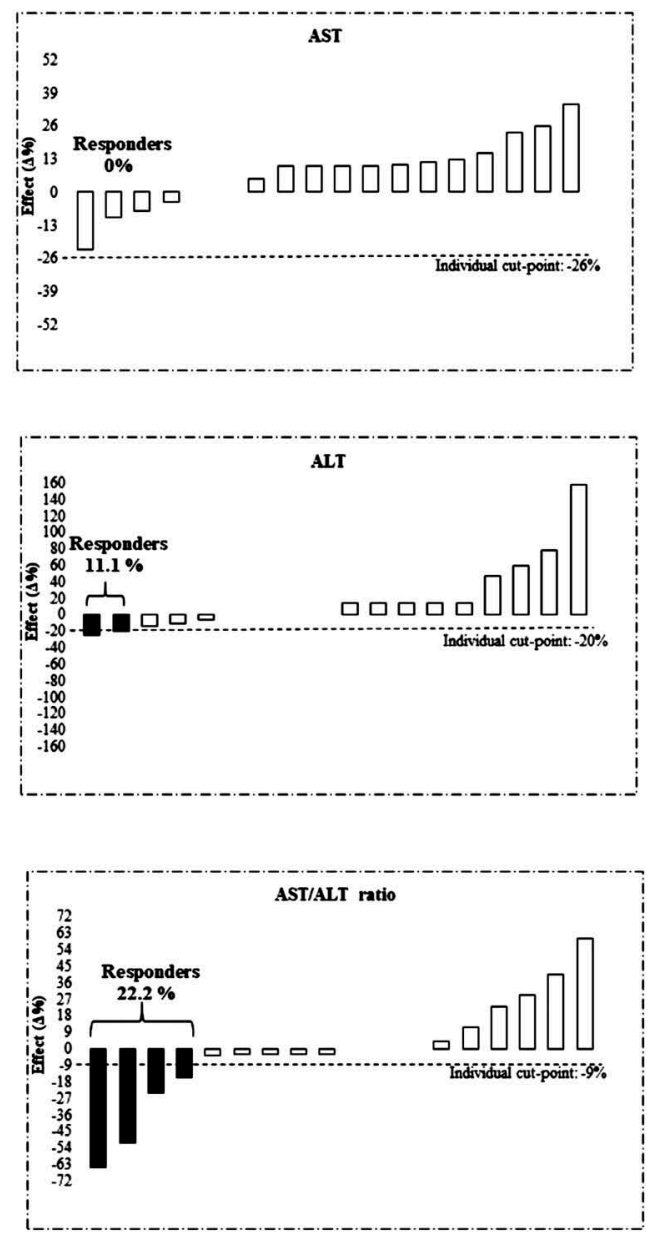

Intervention Group
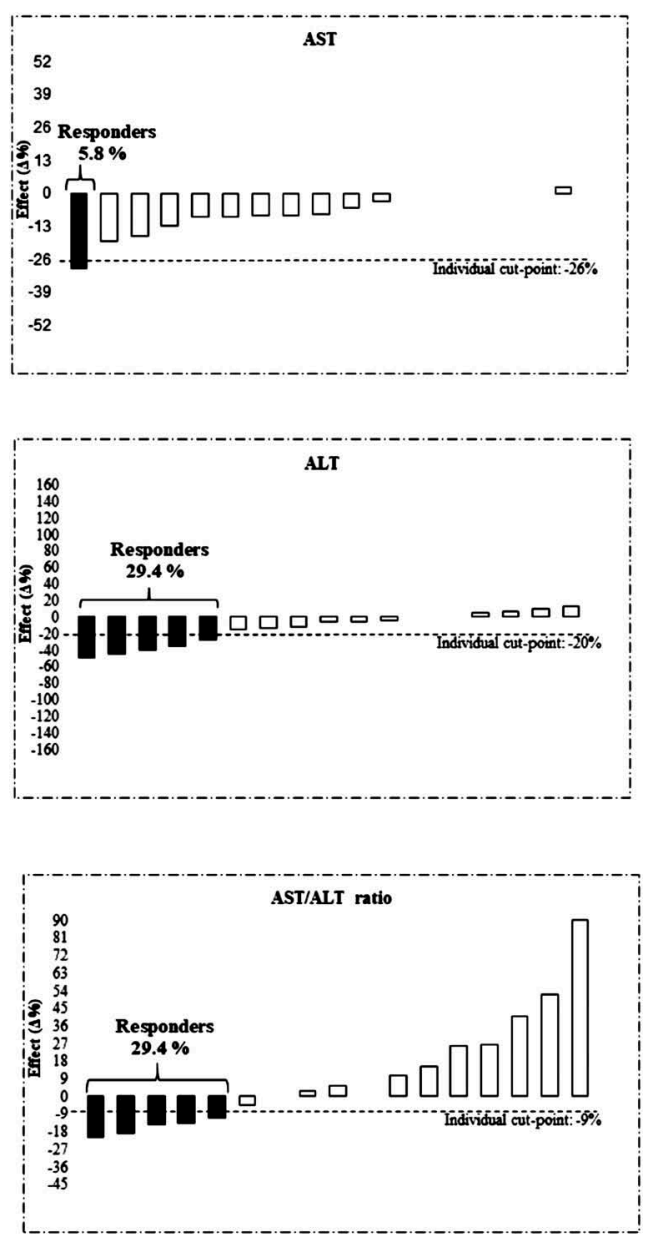

Figure 4. I Prevalence of responders in intervention and control group after a multicomponent intervention for aspartate aminotransferase (AST), alanine aminotransferase (ALT) and AST/ALT ratio. (CG $n=18$ and IG $n=17$ ). *difference in the prevalence ratio between groups.

and resistance training, it was found a prevalence of nonresponders in body fatness of $33.3 \%$ and $70.5 \%$ in woman, respectively (Álvarez et al., 2017a).

It is important to notice that the main responsible for interindividual variability are genetic and environmental factors, such as different modes of exercise or health status (Bauman et al., 2012; Ridgers, Stratton, \& Fairclough, 2011). Nevertheless, many of these factors have not been explored, in either adult and children (Mann, Lamberts, \& Lambert, 2014). Also, some individuals experience adverse responses when exposed to regular exercise, but the causes of this phenomenon are still unknown (Bouchard et al., 2012). Thus, it is not known which mode of training may induce an increased or decreased amount of responders after interventions. Such information could be useful to develop physical activity programs taken into consideration the individual responsiveness, in order to be more effective in promoting health among children and adolescents with overweight/obesity.

The strengths of this study were that we included both the effect of a multicomponent intervention in a "mean term" as well as interindividual variability, which has been less investigated in relation to CMRF among children and adolescents with overweight/obesity. Otherwise, some limitation should be mentioned, such as the sample size and the quasi-experimental design of the intervention that require caution regarding the extrapolation of these results, although we carried out the study with children and adolescents with overweight/obesity and data regarding specific intervention among those population should be enhanced. The intervention covered a limited time period (12 weeks) and, therefore, it would be interesting knowing whether a long-term program would result in continued improvement. Also, the lack of a standardised method to define the cut-point of responders could be considered a limitation. However, as mentioned above, the cut-points were established considering studies with similar characteristics to our study.

\section{Conclusion}

Our results suggest a positive effect of a multicomponent intervention including exercise training, nutritional education and parental support on CMRF of children and adolescents with overweight/obesity. In addition, there was a high prevalence of responders in most of the CMRF evaluated (body fatness, HOMA-IR, HDL-C, LDL-C, TC and ALT), which we consider 
important and clinically relevant results. Thus, these data reinforce the program effectiveness and implementation of this model of intervention might be considered by schools and policymakers, since its benefits seem to be established.

\section{Disclosure statement}

No potential conflict of interest was reported by the authors.

\section{Funding}

This work was supported by the National Council for Scientific and Technological Development (CNPQ) [ID: 477893/2013-9], Coordination for the Improvement of Higher Education Personnel (CAPES) and Foundation for Science and Technology (FCT), Portugal: [SFRH/BSAB/142983/2018 and UID/DTP/00617/201].

\section{ORCID}

Vanilson Batista Lemes (iD http://orcid.org/0000-0003-3298-4449 Jorge Mota (iD) http://orcid.org/0000-0001-7571-9181

\section{References}

Africa, J. A., Newton, K. P., \& Schwimmer, J. B. (2016). Lifestyle interventions including nutrition, exercise, and supplements for nonalcoholic fatty liver disease in children. Digestive Diseases and Sciences, 61(5), 1375-1386.

Aires, L., Silva, G., Martins, C., Marques, E., Lagoa, M. J., Ribeiro, J. C., ... Mota, J. (2016). Exercise intervention and cardiovascular risk factors in obese children. Comparison between obese youngsters taking part in a physical activity school-based programme with and without individualised diet counselling: The ACORDA project. Annals of Human Biology, 43(3), 183-190.

Alkhater, S. A. (2015). Paediatric non-alcoholic fatty liver disease: An overview. Obesity Reviews, 16(5), 393-405.

Alkhouri, N., \& McCullough, A. J. (2012). Noninvasive diagnosis of NASH and liver fibrosis within the spectrum of NAFLD. Journal of Gastroenterology and Hepatology, 8(10), 661-668.

Álvarez, C., Ramírez-Campillo, R., \& Ramírez-vélez, R. (2017c). Prevalence of non-responders for glucose control markers after 10 weeks of high-intensity interval training in adult women with higher and lower insulin resistance. Frontiers in Physiology, 8(July). doi:10.3389/ fphys.2017.00479

Alvarez, C., Ramírez-Campillo, R., Ramírez-Vélez, R., \& Izquierdo, M. (2017b). Effects of 6-weeks high-intensity interval training in schoolchildren with insulin resistance: Influence of biological maturation on metabolic, body composition, cardiovascular and performance non-responses. Front Physiol, 8, 444.

Álvarez, C., Ramírez-Campillo, R., Ramírez-Vélez, R., \& Izquierdo, M. (2017a). Effects and prevalence of nonresponders after 12 weeks of high-intensity interval or resistance training in women with insulin resistance: A randomized trial. Journal of Applied Physiology, 122(4), 985-996.

Álvarez, C., Ramírez-Campillo, R., Ramírez-Vélez, R., Martínez, C., CastroSepúlveda, M., Alonso-Martínez, A., \& Izquierdo, M. (2018). Metabolic effects of resistance or high-intensity interval training among glycemic control-nonresponsive children with insulin resistance. International Journal of Obesity, 42(1), 79-87.

Bauman, A. E., Reis, R. S., Sallis, J. F., Wells, J. C., Loos, R. J. F., Martin, B. W., ... Group, W. (2012). Physical activity 2 correlates of physical activity : Why are some people physically active and others not? The Lancet, 380(9838), 258-271.

Bonafiglia, J. T., Rotundo, M. P., Whittall, J. P., Scribbans, T. D., Graham, R. B., \& Gurd, B. J. (2016). Inter-individual variability in the adaptive responses to endurance and sprint interval training: A randomized crossover study. PLOS ONE, 11(12), e0167790.

Bouchard, C., Blair, S. N., Church, T. S., Earnest, C. P., Hagberg, J. M., Jenkins, N. T., ... Rankinen, T. (2012). Adverse metabolic response to regular exercise : Is it a rare or common occurrence ? 7(5). doi:10.1371/ journal.pone.0037887.

Bouchard, C., \& Rankinen, T. (2001). Individual differences in response to regular physical activity. Medicine Science in Sports and Exercise, 33(6), 446-451.

Chang, C., Jain, D., Lin, M., Zhao, J., Ho, L., \& Juan, C. (2015). Evidence in obese children: Contribution of hyperlipidemia, obesity-inflammation, and insulin sensitivity. Plos One, 10(5). doi:10.1371/journal.pone.0125935

Cole, T. J., Bellizzi, M. C., Flegal, K. M., \& Dietz, W. H. (2000). Establishing a standard definition for child overweight and obesity worldwide: International survey. BMJ, 320(7244), 1240-1243.

Draijer, L. G., Feddouli, S., Bohte, A. E., Slootweg, O. B., Rijcken, T. H. P., Benninga, M. A., ... Koot, B. G. P. (2019). Comparison of diagnostic accuracy of screening tests ALT and ultrasound for pediatric non-alcoholic fatty liver disease. European Journal of Pediatrics, 178(6), 863-870.

Ells, L. J., Rees, K., Brown, T., Mead, E., Al-Khudairy, L., Azevedo, L., \& Demaio, A. (2018). Interventions for treating children and adolescents with overweight and obesity: An overview of Cochrane reviews. International Journal of Obesity, 42(11), 1823-1833.

Escalante, Y., Saavedra, J. M., Garcia-Hermoso, A., \& Dominguez, A. M. (2012). Improvement of the lipid profile with exercise in obese children: A systematic review. Preventive Medicine, 54(5), 293-301.

Fang, X., Zuo, J., Zhou, J., Cai, J., Chen, C., Xiang, E., ... Chen, P. (2019). Childhood obesity leads to adult type 2 diabetes and coronary artery diseases. A 2-sample mendelian randomization study. Medicine, 98(32), e16825.

Fedewa, M. V., Gist, N. H., Evans, E. M., \& Dishman, R. K. (2014). Exercise and insulin resistance in youth: A meta-analysis. Pediatrics, 133(1), e163e174.

Flores, L. S., Gaya, A. R., Petersen, R. D. S., \& Gaya, A. (2013). Trends of underweight, overweight, and obesity in Brazilian children and adolescents. Jornal De Pediatria, 89(5), 456-461.

Friedewald, W. T., Levy, R. I., \& Fredrickson, D. S. (1972). Estimation of the concentration of low-density lipoprotein cholesterol in plasma, without use of the preparative ultracentrifuge. Clinical Chemistry, 18(6), 499-502.

Gaya, A. R., Dias, A. F., Lemes, V. B., Gonçalves, J. C., Marques, P. A., Guedes, G., .. Gaya, A. C. A. (2017). Aggregation of risk indicators to cardiometabolic and musculoskeletal health in Brazilian adolescents in the periods 2008/09 and 2013/14. Jornal De Pediatria. doi:10.1016/j. jped.2017.04.006

Hardy, L. L., Mihrshahi, S., Gale, J., Drayton, B. A., Bauman, A., \& Mitchell, J. (2017). 30-year trends in overweight, obesity and waist-to-height ratio by socioeconomic status in Australian children, 1985 to 2015. International Journal of Obesity, 41(1), 76-82.

Hauger, H., Groth, M. V., Ritz, C., Biltoft-Jensen, A., Andersen, R., Dalskov, S. M., ... Damsgaard, C. T. (2016). Socio-economic differences in cardiometabolic risk markers are mediated by diet and body fatness in 8- to 11-year-old Danish children: A cross-sectional study. Public Health Nutrition, 19(12), 2229-2239.

Ho, M., Garnett, S. P., Baur, L., Burrows, T., Stewart, L., Neve, M., \& Collins, C. (2012). Effectiveness of lifestyle interventions in child obesity: systematic review with meta-analysis. Pediatrics, 130(6), 2012.

Hopkins, W. G. (2000). Measures of reliability in sports medicine and science. Sports Medicine (Auckland, N.Z.), 30(1), 1-15.

Karacabey, K. (2009). The effect of exercise on leptin, insulin, cortisol and lipid profiles in obese children. Journal of Internacional Medical Research, 37(5), 1472-1478.

Koot, B. G. P., van der Baan-slootweg, O. H., Tamminga-Smeulders, C. L. J., Rijcken, T. H. P., Korevaar, J. C., van Aalderen, W. M., ... Benninga, M. A. (2011). Lifestyle intervention for non-alcoholic fatty liver disease: Prospective cohort study of its efficacy and factors related to improvement. Archives of Disease in Childhood, 96(7), 669-674.

Kraus, E. (2002). Effects of the amount and intensity of exercise on plasma lipoproteins. N Engl J Med, 347(19), 1483-1492.

Lohman, T. G., Roche, A. F., \& Martorell, R. (1988). Anthropometric standardization reference manual. Champaign, IL: Human Kinetics Books. 
Mann, T. N., Lamberts, R. P., \& Lambert, M. I. (2014). High responders and low responders : Factors associated with individual variation in response to standardized training. Sports Medicine (Auckland, N.Z.), 44(8), 1113-1124.

Matthews, D. R., Hosker, J. P., Rudenski, A. S., Naylor, B. A., Treacher, D. F., \& Turner, R. C. (1985). Homeostasis model assessment: Insulin resistance and beta-cell function from fasting plasma glucose and insulin concentrations in man. Diabetologia, 28(7), 412-419.

Medrano, M., Cadenas-Sanchez, C., Álvarez-Bueno, C., Cavero-Redondo, I., Ruiz, J. R., Ortega, F. B., \& Labayen, I. (2018). Evidence-Based Exercise Recommendations to Reduce Hepatic Fat Content in Youtha Systematic Review and Meta-Analysis. Progress in Cardiovascular Diseases, 61(2), 222-231.

Meyer, A. A., Kundt, G., Lenschow, U., Schuff-werner, P., \& Kienast, W. (2006) Improvement of Early vascular changes and cardiovascular risk factors in obese children after a six-month exercise program. 48(9). doi:10.1016/j. jacc.2006.07.035.

Montero, D., \& Lundby, C. (2017). Refuting the myth of non-response to exercise training: 'non-responders' do respond to higher dose of training. Journal of Physiology, 595(11), 3377-3387.

Nascimento, H., Costa, E., Rocha, S., Lucena, C., Rocha-Pereira, P., Rêgo, C., .. Belo, L. (2014). Adiponectin and markers of metabolic syndrome in obese children and adolescents: Impact of 8-mo regular physical exercise program. Pediatric Research, 6(2), 1-7.

Reinehr, T., Schaefer, A., Winkel, K., Finne, E., Toschke, A. M., \& Kolip, P. (2010). An effective lifestyle intervention in overweight children: Findings from a randomized controlled trial on "Obeldicks light.". Clinical Nutrition, 29(3), 331-336.

Resaland, G., Nilsen, A., Bartholomew, J., Andersen, L., \& Anderssen, S. (2018). The effect of a two year school-based daily physical activity intervention on a clustered CVD risk factor score - The Sogndal school-intervention study. Scand J Med Sci Sports, 28(3), 1027-1035.

Ridgers, N., Stratton, G., \& Fairclough, S. J. (2011). Associations between selected demographic, biological, school environmental and physical education based correlates, and adolescent physical activity. Pediatr Exerc Sci, 23(1), 61-71.
Sahoo, K., Sahoo, B., Choudhury, A. K., Sofi, N. H., Kumar, R., \& Bhadoria, A. J. (2015). Childhood obesity: Causes and consequences. Journal of Family Medicine and Primary Care, 4(2), 187-192.

Silveira, D. S., Lemos, L. F. G. B. F., Tassitano, R. M., Cattuzzo, M. T., Feitoza, A. H. P., Aires, L. M. S. M. C., ... Martins, C. M. L. (2018). Effect of a pilot multi-component intervention on motor performance and metabolic risks in overweight/obese youth. Journal of Sport Science, 36(20), 2317-2326.

Talayero, B. G., \& Sacks, F. M. (2011). The role of triglycerides in atherosclerosis. Current Cardiology Reports, 13(6), 544-552.

Toth, P. P., Barter, P. J., Rosenson, R. S., Boden, W. E., Chapman, M. J., Cuchel, M., ... Rader, D. J. (2013). High-density lipoproteins: A consensus statement from the national lipid association. Journal of Clinical Lipidology, 7(5), 484-525.

US Department of Health and Human Services. (1996). NHANES III. anthropometric procedures. Washington.

Vos, M. B., Abrams, S. H., Barlow, S. E., Caprio, S., Daniels, S. R., Kohli, R., ... Xanthakos, S. A. (2017). NASPGHAN clinical practice guideline for the diagnosis and treatment of nonalcoholic fatty liver disease in children: Recommendations from the expert committee on NAFLD (ECON) and the North American society of pediatric gastroenterology, hepatology and nutrition. Journal of Pediatric Gastroenterology and Nutrition, 64(2), 319-334.

Wang, Y., \& Xu, D. (2017). Effects of aerobic exercise on lipids and lipoproteins. Lipids in Health and Disease, 16(1), 1-8.

Weineck, J. (2005). Biologia do Esporte. São Paulo: Manole.

World Health Organization. (2017). Levels and trends in child malnutrition. UNICEF/WHO/world bank group joint malnutrition estimates. New York/ Geneva/Washington DC: Author.

World Medical Association. (2013). Declaration of helsinki ethical principles for medical research involving human subjects.

Yin, J., Li, M., Xu, L., Wang, Y., Cheng, H., Zhao, X., \& Mi, J. (2013). Insulin resistance determined by homeostasis model assessment (HOMA) and associations with metabolic syndrome among Chinese children and teenagers. Diabetology \& Metabolic Syndrome, 5(1), 71. 